\title{
Alternative integration of ultralow-k dielectrics by template replacement approach
}

\author{
L. Zhang ${ }^{1,2}$, J.-F. de Marneffe ${ }^{1}$, N. Heylen ${ }^{1}$, G. Murdoch ${ }^{1}$, Z. Tokei ${ }^{1}$, \\ J. Boemmels ${ }^{1}$, S. De Gendt ${ }^{1,2}$ and M. R. Baklanov ${ }^{1}$ \\ ${ }^{1}$ IMEC, Kapeldreef 75, 3001 Heverlee, Belgium \\ ${ }^{2}$ Department of Chemistry, Katholieke Universiteit Leuven, 3000 Leuven, Belgium \\ Email: Liping.Zhang@imec.be
}

\begin{abstract}
Replacement of sacrificial template by ultralow-k dielectric was studied as an alternative integration approach for $\mathrm{Cu} /$ low-k interconnect. Metallization structure was first formed by patterning a template material. After template removal, a spin-on porous low-k was deposited on the metal lines. Then, planarization of the excess low-k was performed by CMP. The proposed approach does solve the two major challenges in conventional $\mathrm{Cu} /$ low-k damascene integration approach: low-k plasma damage and metal penetration during barrier deposition on porous structures.
\end{abstract}

\section{Introduction}

$\mathrm{Cu} /$ low-k replaced traditional $\mathrm{Al} / \mathrm{SiO}_{2}$ in order to reduce resistance-capacitance signal delay and power dissipation. The damascene approach was introduced, due to the difficulty to etch Copper. However, traditional damascene approach is becoming increasingly difficult as critical dimension continuously scale down [1]. For low$\mathrm{k}$ with large pore size and open porosity, patterning with plasma is facing challenge of severe plasma induced damage [2]. Larger pore size and post etch roughness also lead to penetration of barrier, causing reliability issue.

In order to overcome these challenges, we studied an alternative integration which essentially rely on the replacement of a sacrificial template by spin-on ultralow-k material after metallization. Trench/via was first formed by conventional Litho-Etch patterning steps with sacrificial template (Fig.1). After metallization, including barrier deposition, $\mathrm{Cu}$ plating and CMP, the template layer was stripped by downstream plasma (DSP). By this way, metal lines were formed without direct plasma etching of $\mathrm{Cu}$. By spin coating followed by hard bake, a porous low$\mathrm{k}$ dielectric encapsulated the metal gap structure. Fig.1 describes the template replacement approach. The three major steps, metallization with template, low-k gap-filling and planarization, will be discussed as main part of this paper.

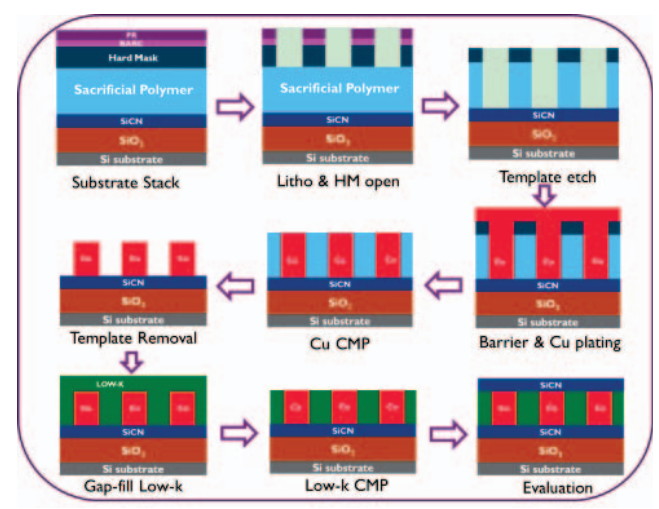

FIG. 1. Schematic shows single damascene $\mathrm{Cu} /$ low-k interconnect process flow with low-k replacement approach.

\section{Experimental and Discussion}

Metallization with template: The first stage is the definition of trench/via structure in the template material. This material should be compatible with plasma patterning at small dimensions and high aspect ratio structure, sustain the thermal budget required by metallization steps and CMP. In the present work, an amorphous carbon $(\alpha-\mathrm{C})$ layer was deposited by CVD at $400^{\circ} \mathrm{C}$. High thermal stability and mechanical strength offered compatibility with BEOL process conditions.

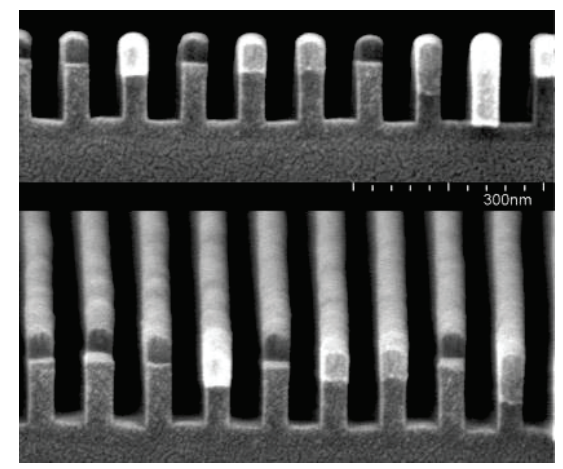

FIG. 2. SEM cross section and titled view of template lines after etching with $\mathrm{N}_{2}$ based plasma. 
With both silica based or metallic hard mask, excellent etch selectivity and line roughness were obtained by $\mathrm{N}_{2} / \mathrm{H}_{2}$ based $\mathrm{CCP}$ gas discharges. Fig.2 shows trenches patterned with a $\mathrm{N}_{2} / \mathrm{H}_{2}-$ based plasma. Compared with porous low-k in case of normal damascene process, surface roughness (RMS $0.4 \mathrm{~nm}$ ) is greatly improved. Post etch surface also showed good adhesion to $\mathrm{TaN} / \mathrm{Ta}$ barrier. This is essential to get a continuous metal barrier.

Once the metal structure was formed, this sacrificial material must be stripped away completely without $\mathrm{Cu}$ damage. A $\mathrm{H}_{2}$-based downstream plasma was used to strip the $\alpha-C$ template in between $\mathrm{Cu}$ lines. The $\mathrm{H}_{2}$ DSP contains no energetic ions or reactive species $(\mathrm{F}$, $\mathrm{Cl}$ or $\mathrm{O}$ ), and hence cause no physical sputtering or chemical corrosion to metallization structure. It is also isotropic therefore compatible with dualdamascene structures. $35 \mathrm{~nm}$ metal lines $(\mathrm{Cu}$ and barrier) at $90 \mathrm{~nm}$ pitch were obtained after $\mathrm{Cu}$ CMP. CDSEM measurement showed no change after template removal, confirming no damage to metal lines. Finally, high aspect ratio $\mathrm{Cu}$ trench was formed with continuous thin barrier coated on sidewall, as shown in Fig.3.

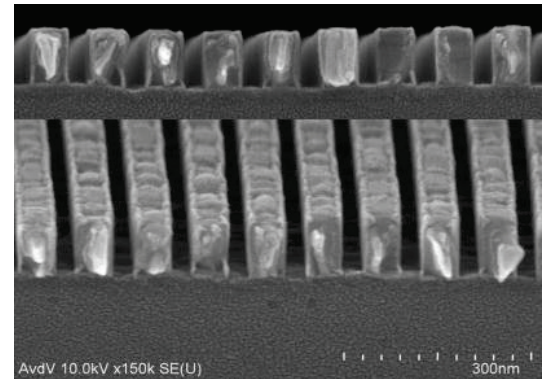

FIG. 3. Schematic shows SEM cross section and titled view of $\mathrm{Cu}$ lines after metallization and template removal.

Gap-filling porous low-k: By spin-on method, a solution containing both matrix and porogen precursors was coated onto wafer with patterned metal structure. Surfactant compositions in the sol enable a good wettability of the metal barrier surface (TaN in this case). The sol fills the trench without void formation. After coating, wafers went for soft bake and hard bake, in order to remove solvent and porogen, respectively. A short time exposure with UV lamp was applied to remove porogen residues. Fig. 4 shows the change of thickness and RI of low-k films, which were measured by Spectroscopic Ellipsometry (SE) on a $100 \times 100 \mathrm{um}^{2}$ pad. Although most of template is burned out by hard baking, UV cure helps to remove template residues [3]. This decreased further refractive index. Porosity was increased and lower k-value could be obtained.
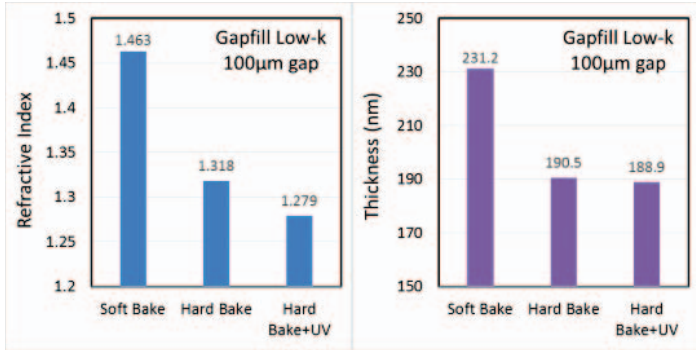

FIG. 4. Refractive Index and film Thickness change with baking and UV curing to make porous low-k.

Finally, after low-k gap filling the morphology was inspected by cross-section SEM, as shown in Fig.5. After coating and template removal, the gaps in between $\mathrm{Cu}$ lines are completely filled by the porous low-k. Film shrinkage up to $18 \%$, as observed in 100 micrometer pad, doesn't lead to delamination at the interface between the low-k and the barrier layer.

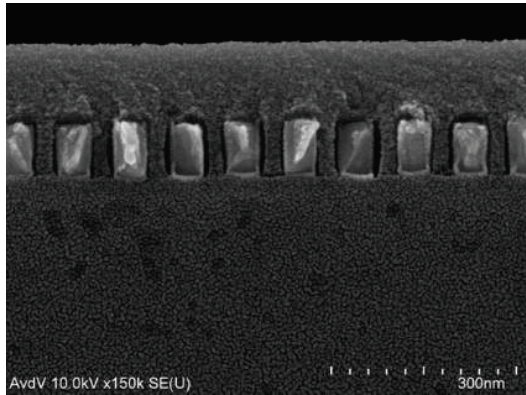

FIG. 5. Schematic shows SEM cross section after low-k gapfill and porogen burn-out. Note that the small gap between low-k and metal lines is artifact of low-k shrinkage caused by high voltage e-beam scanning during SEM measurement.

Low-k planarization: Fig.5 shows also that approximately $120 \mathrm{~nm}$ low-k remained on top of metal layer. Planarization of spin coated low-k on uneven surface requires a CMP-like approach. Direct low-k CMP was developed by using an oxide slurry. However, $\mathrm{k}$ value extracted with blanket film degraded from 2.33 to 2.53 .

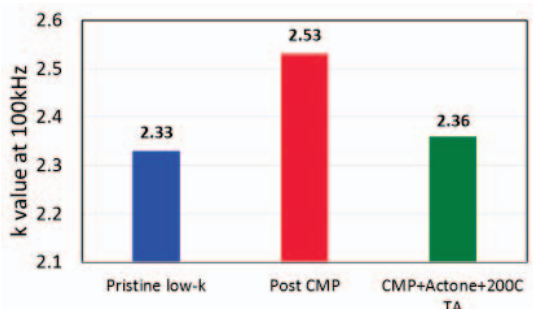

FIG. 6. $\mathrm{k}$ value degradation and restoration. Note that it is extracted by CV measurement at $100 \mathrm{kHz}$ with blanket films. 
FTIR (Fig.7) shows that CMP actually doesn't lead to loss of $\mathrm{Si}-\mathrm{CH}_{3}$ groups at $1276 \mathrm{~cm}^{-1}$, and no deformation of Si-O-Si peaks. This means there is no modification of chemical composition as in case of plasma exposure [4]. Extra peaks increased at $2900 \mathrm{~cm}^{-1}$ indicate that organic composition (mostly surfactant in slurry) penetrates into the porous structure. A post-CMP cleaning sequence was thus developed to remove slurry residues and restore the $\mathrm{k}$ value. Rinse with an organic solvent followed by a soft bake removed extra FTIR peaks and pristine k value was recovered.
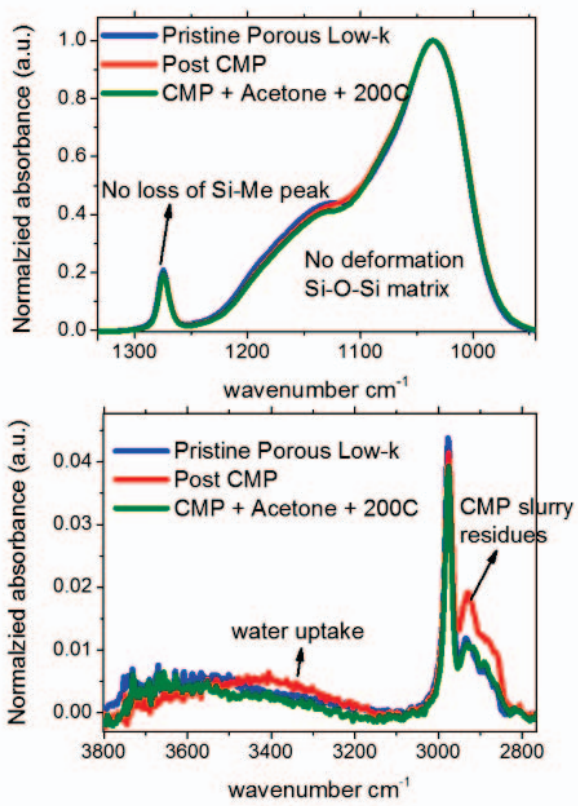

FIG. 7. FTIR spectra shows chemical composition change after CMP and post CMP repair.

CMP and post-clean were transferred to patterned wafers. No delamination or defects were observed after CMP down to the $\mathrm{Cu}$, as observed by $\mathrm{CD}$ SEM. This also indicate a good adhesion between the gap-fill low-k and the metal barrier. Fig.8 shows the change of line morphology at three different stages: Post $\mathrm{Cu}$ CMP, Post template removal and Post low-k CMP. Note that low-k CMP did not reach the metal layer in the last image. The thin residual low-k cap scatters the ebeam, blurring the image.

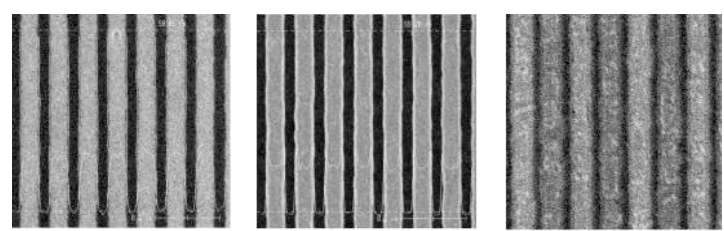

FIG. 8. Top-Down SEM shows CD change. (a) Post $\mathrm{Cu}$ CMP, (b) Post H2 plasma stripping, (c) Post low-k CMP.

\section{Conclusion}

Using a CVD amorphous carbon as a template, the metal interconnect structure has been formed with high aspect ratio and low edge roughness. The a$\mathrm{C}$ provide a non-porous surface with good adhesion to the barrier. After $\mathrm{Cu} \mathrm{CMP}$, an $\mathrm{H}_{2}$ based downstream plasma completely remove the $\alpha-C$ without damage to the metal layer.

Spin-on porous organosilicate low-k was used to fill the trenches formed in the metal layer. After baking and UV curing, no gap or delamination was observed.

Finally, the planarization of excess low-k on top of metal layer was achieved by direct CMP, causing no damage/modification to the porous low-k matrix. Although penetration of slurry residues lead to $\mathrm{k}$ degradation, it can be easily removed by post CMP cleaning.

The proposed method eliminates plasma-induced low-k damage, as well as barrier penetration issues.

\section{Acknowledgements}

The authors would like to thank Hash Pakbaz from SBA Materials for providing sols for low-k deposition and the imec X-SEM group for cross section measurement.

\section{Reference}

${ }^{[1]}$ I.S. Devised, I. For, T. Assessment, I.S. Without, R. To, C. Considerations, P. To, I. Products, and O.R. Equipment, ITRS (2011).

[2] M.R. Baklanov, J.-F. de Marneffe, D. Shamiryan, A.M. Urbanowicz, H. Shi, T. V. Rakhimova, H. Huang, and P.S. Ho, J. Appl. Phys. 113, 041101 (2013).

[3] A.M. Urbanowicz, K. Vanstreels, P. Verdonck, D. Shamiryan, S. De Gendt, and M.R. Baklanov, J. Appl. Phys., 107, 104122 (2010).

${ }^{[4]}$ T. Oszinda, V. Beyer, M. Schaller, D. Fischer, C. Bartsch, and S.E. Schulz, J. Vac. Sci. Technol. B . 27, 521 (2009). 
